\title{
Effect of Whole-Body Vibration Exercise on Balance in Women with Fibromyalgia Syndrome: A Randomized Controlled Trial
}

\author{
Borja Sañudo Corrales, PhD, Moises de Hoyo, MSc, Luis Carrasco, PhD, \\ Cleofás Rodríguez-Blanco, PhD, Ángel Oliva-Pascual-Vaca, PhD, ${ }^{2}$ and Joseph G. McVeigh, $\mathrm{PhD}^{3}$
}

\begin{abstract}
Objectives: This study evaluated the effectiveness of a 6-week "usual care" exercise program supplemented with whole-body vibration (WBV) to improve balance and strength in women with fibromyalgia (FM).

Design: This was a randomized controlled study.

Settings: The setting was a physical therapy department in an academic setting.

Subjects: The subjects were 30 postmenopausal women with FM (age: $59 \pm 7.90$ years).

Interventions: Subjects were randomized into one of two groups: an experimental group (EG: $n=15)$, which combined exercise training ( 2 days a week) with 3 days of WBV, and a control group (CG: $n=15)$, who performed the same exercise training program (2 days a week) but without WBV.

Outcome measures: Balance and muscle strength were measured at baseline and after the 6-week intervention. Results: Significant differences were found $(p<0.05)$ between the study groups for the Medio-Lateral Stability Index (MLSI), when patients were assessed with their eyes open and closed. The effect size of the improvement was large with eyes closed $\left(R^{2}=0.260\right)$ and moderate when the eyes were open $\left(R^{2}=0.047\right)$. However, no significant differences were found $(p>0.05)$ between the study groups for other outcomes.

Conclusions: Women with FM may increase their MLSI by engaging in a 6-week traditional exercise program with supplementary WBV. This may have implications for falls prevention in this patient group.
\end{abstract}

\section{Introduction}

$\mathbf{F}$ IBROMYALGIA SYNDROME (FM) is a perplexing yet common pain syndrome. Patients present with chronic widespread pain, and tenderness in at least 11 of 18 specific tender points, as defined by the American College of Rheumatology (ACR) criteria. ${ }^{1}$ Many patients with FM also suffer from sleep disorders, ${ }^{2}$ and physical problems, such as reduced muscle strength, ${ }^{3,4}$ muscle stiffness, ${ }^{5-7}$ and bodily fatigue. ${ }^{6,8}$ It has also been reported recently that patients with FM have problems with motor control ${ }^{9}$ and postural balance. ${ }^{10} \mathrm{Im}$ portantly, it has also been noted that people with FM limit their physical activity due to fear of falling, muscular weakness, and impaired balance. ${ }^{11}$ Indeed, a number studies have shown that there is a significant correlation between functional disability and postural balance in patients with FM and in those suffering from other rheumatic disorders such as rheumatoid arthritis. ${ }^{12,13}$ The combined impact of these problems on patients' function and quality of life is well documented. . $^{511,14}$

Balance is a neural function, integrated by a complex network of proprioceptors and controlled responses, $4,15,16$ which may be altered in FM. Indeed, there is evidence of diminished postural balance in patients with rheumatic syndromes including FM. ${ }^{11,13,16,17}$ It is suggested that this impaired balance in FM may be due to an altered perception or an altered interpretation of audio-vestibular inputs. ${ }^{18}$ Furthermore, there is evidence of increased muscle tension in postural muscles in patients with FM, which results in difficulty in achieving relaxation and a tendency to fatigue. ${ }^{19}$ Additional evidence of balance dysfunction in FM was reported by Jones et al., ${ }^{17}$ who found that patients with FM have significantly worse balance $(F(1)=10.62, p=0.002)$ and greater fall frequency compared to age-matched controls. In

\footnotetext{
${ }^{1}$ Department of Physical Education and Sport, Faculty of Health Sciences, University of Seville, Seville, Spain.

${ }^{2}$ Department of Physiotherapy, University of Seville, Faculty of Health Sciences, Seville, Spain.

${ }^{3}$ Health and Rehabilitation Sciences Research Institute, School of Health Sciences, University of Ulster, Jordanstown, Northern Ireland.
} 
a large Internet survey $(n=2596)$, people with self-reported FM also identified balance problems as one of their most common symptoms after pain-related difficulties. ${ }^{20}$ If it is the case that patients with FM have impaired balance reactions and are at greater risk of falls, then an important treatment goal would be to develop the proprioceptive capabilities of patients by stimulating the neuromuscular system, which may assist in preventing falls. ${ }^{21-23}$

Several studies have shown that exercise training can improve balance in older people ${ }^{24,25}$ and in recent years a number of authors have demonstrated that whole-body vibration (WBV) training has been associated with increased lower limb muscle strength in various musculoskeletal conditions including FM. ${ }^{23,25-27}$ However, despite the benefits in muscular performance, the impact of WBV on balance is still uncertain. ${ }^{28}$ Some authors have reported an improvement in balance after 6 or 8 weeks of WBV training combined with physical therapy in elderly people. ${ }^{25,29,30} \mathrm{~A}$ recent study also reported that twice-weekly mixed WBV exercises and landbased exercise lowered pain scores and fatigue ratings in individuals with FM. ${ }^{7}$ The group in the current study has also found improvements in functional capacity and quality of life with a 6-week traditional exercise program supplemented with WBV. ${ }^{31}$ To the authors' knowledge, only one study has previously assessed the feasibility and efficacy of intensive WBV for balance in patients with FM; these authors reported that WBV significantly improved dynamic balance in those with FM. ${ }^{32}$

The aim of this study was to determine whether implementation of a training protocol using WBV platforms would alter lower limb muscle strength and postural balance in patients with FM.

\section{Materials and Methods}

Participants in this randomized clinical trial were a convenience sample of 30 postmenopausal women with a confirmed diagnosis of FM, based on the ACR criteria. ${ }^{1}$ Participants were recruited from either rheumatology clinics or FM patient support groups in Seville, Spain. Potential participants were contacted by a physician and after initial screening for inclusion participants were invited for an interview where informed written consent was obtained.

Participants were excluded from the study if they had any concomitant medical illness that might be exacerbated by the physical activity: for example, inflammatory diseases, degenerative joint diseases, and respiratory or cardiovascular diseases. People were also excluded from the study if they were currently under the care of a psychiatrist. Subjects who were performing structured physical activity for at least 2 days per week or had attended for psychologic therapy in the previous 6 months were also excluded.

Forty (40) participants were assessed for eligibility to participate in the study, and 30 patients who met the inclusion criteria were randomly allocated into one of two groups: The intervention group (EG) which consisted of combined exercise training with WBV $(n=15)$, or control group (CG), who performed the same exercise training program but without vibration training $(n=15)$ for 6 weeks. Randomization was based on a computer-generated random number table and group allocation was conducted by DG who was unaware of the randomization sequence, and was not involved in the day to day running of the trial. The study was approved by the ethics committee of the University of Seville and also complied with the Helsinki Ethical Principles for Medical Research Involving Human Patients and its subsequent modifications.

\section{Procedures}

Assessment of all outcomes was undertaken at baseline (T1) and after the 6-week intervention (T2). The intervention for both groups consisted of two exercise training sessions per week and three additional sessions with WBV for the EG group only. Training sessions included a combination of aerobic and muscle strength training exercises, including 10minute warm-up, 10-15 minutes of aerobic exercise at 65\%$70 \%$ maximum heart rate, $15-20$ minutes of muscle training (eight exercises, each station 1 set of $8-10$ reps with $1-3 \mathrm{~kg}$ ) and finally, 10 minutes of flexibility training (eight or nine exercises, each station 1 set of 3 repetitions, holding the stretched position for 30 seconds). Heart rate was recorded at each session to ensure that each participant exercised at the appropriate intensity (Appendix 1).

The Galileo Fitness ${ }^{\circledR}$ platform (Novotech, Germany) was used for the vibration protocol. Participants were asked to stand on the vibration platform with both knees in $120^{\circ}$ isometric knee flexion (half squatting position), as measured by a goniometer. Participants were then submitted to a vibration frequency of $20 \mathrm{~Hz}$ and variable amplitude of 2-3 mm. Each session was three sets of 45 seconds with 120second recovery between sets (amplitude $=3 \mathrm{~mm}$ ) and four sets of 15 seconds. The first three sets were performed with participants standing with both feet on the platform for 45 seconds, with vibration set at a frequency of $20 \mathrm{~Hz}$ (amplitude $=3 \mathrm{~mm}$ ). After 120-second recovery between sets, participants then completed four sets of unilateral (i.e., single leg) static squats at a frequency of $20 \mathrm{~Hz}$ (amplitude $=2 \mathrm{~mm}$ ). Participants completed 15 seconds of the exercise with the right leg and then immediately completed 15 seconds on the left leg, and this was considered one set. The intervention lasted for 6 weeks.

\section{Outcome measures}

Participants attended the Physical Education and Sport Laboratory, University of Seville for initial assessment (T1), where baseline demographic information and clinical details were collected.

\section{Assessment of knee extensor muscle strength}

In order to determine the maximal knee extensor strength, participants were asked to perform a half squat in front of a multipower machine with guided weight. Participants were asked to bend their knees to $120^{\circ}$ flexion and grasp the bar at shoulder width. Participants then had to extend their knees with their arms fully extended (avoiding shoulder movement and elbow flexion) and maintain the trunk vertically. The test was performed 3 times with 30-second recovery between each attempt. The force produced (newtons) during the fastest repetition was used for analysis by the system of measurement of dynamic forces T-FORCE system ${ }^{\circledR}$ (Ergotech Consulting, Spain). 


\section{WHOLE BODY VIBRATION TRAINING IN FM}

\section{Balance assessment}

Balance assessments were carried out using a Biodex
Stability System (BSS; Biodex, Inc., Shirley, NY; Fig. 1) which consists of a dynamic balance platform that allows movements around the anterior-posterior (AP) and mediolateral (ML) axes simultaneously. The BSS device is interfaced with its own software (Biodex 3.1, Biodex Medical Systems, Shirley, NY) that enables objective assessment of balance. ${ }^{12,33}$ The BSS measures, in degrees, the tilt about each axis during dynamic conditions and calculates a MedioLateral Stability Index (MLSI), an Anterior-Posterior Stability Index (APSI), and an Overall Stability Index, which is a composite of the MLSI and the APSI. ${ }^{34}$ The BSS also calculates the average position for the patient during all motions throughout the test. This is referred to as the Mean Deflection (MD).The Anterior-Posterior Mean Deflection (APMD) and the Medio-Lateral Mean Deflection (MLMD) were included as dependent variables. A higher score in all these outcomes indicates poorer balance.

The BSS device has eight levels of stability, levels 1-8. It is more difficult to maintain balance at level 1 than at level 8 . To determine the appropriate level of stability to use in the study, a pilot study $(n=12)$ was conducted prior to the main clinical trial. Those in the pilot study met the same inclusion criteria for the main clinical trial (mean [standard deviation; SD] age: 58.8 (5.2) years). Women with FM expressed difficulty maintaining their balance, and slight muscle discom-

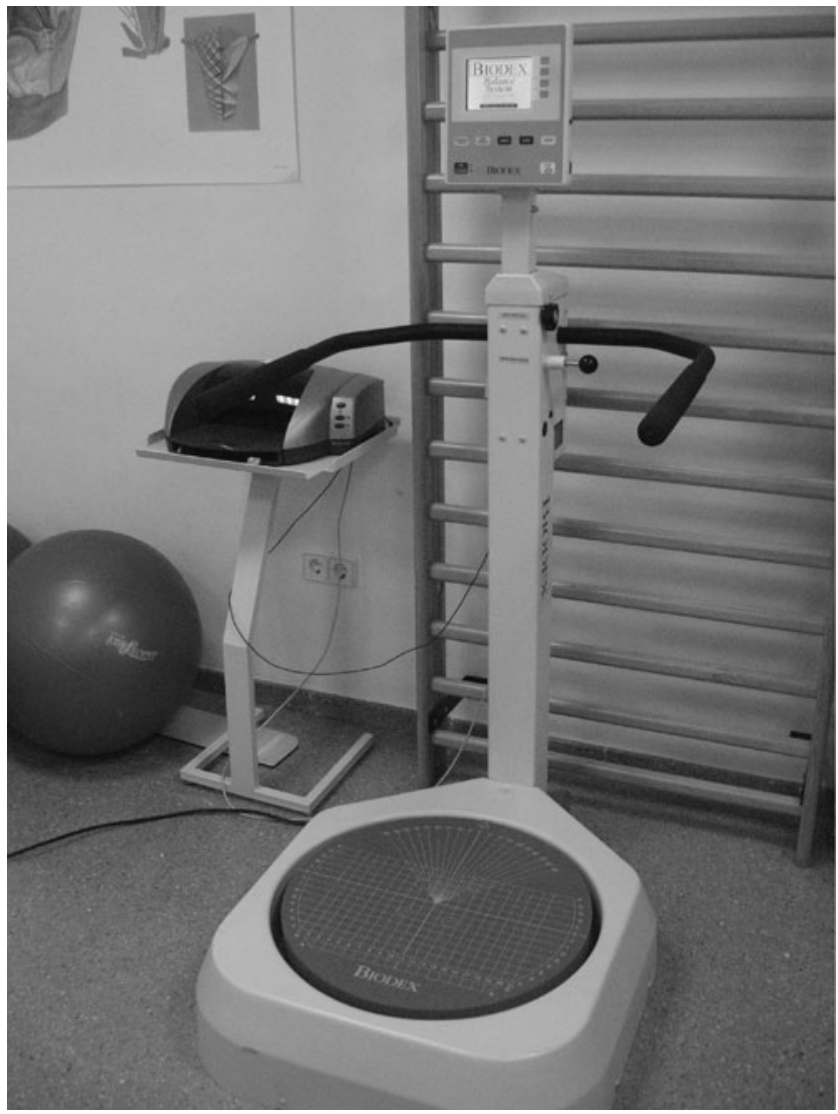

FIG. 1. Biodex Stability System (BSS, Biodex, Inc., Shirley, NY). fort, at stability levels < 5 ; consequently, all measurements were performed at the fifth level of stability.

In order to assess balance, patients were asked to stand on the BSS device for 1 minute. During the assessment period, the platform began to freely move and simultaneously calculate the degree of tilt about both axes (AP and ML). All postural balance measurements were repeated 3 times and the mean was used for the analysis. The tests were performed in two different phases; in the first phase, patients kept their eyes open (visual control) and in the second phase, participants kept their eyes closed.

\section{Statistical analysis}

The normality of the data was calculated using the Kolmogorov-Smirnov test, and the $\chi^{2}$ test was used to compare clinical categorical variables between groups. Baseline features were compared between groups using the independent $t$ tests for continuous data, and the $\chi^{2}$ test of independence for categorical data. An analysis of covariance was used to assess differences between groups with the pre-intervention value used as covariant, group as the fixed/intergroup pfactor, and the postintervention value as dependent variable.

Balance assessment has an intrinsic variability; therefore, when assessing balance, the standard error of the mean (SEM) was calculated. Additionally pre-intervention data of the whole sample (SD/ $\sqrt{ }$ sample size) for all the variables was considered in order to determine whether group differences could be due to an inherent error in the balance data (Table 1).

The statistical analysis was conducted at a $95 \%$ confidence level. A $p$-value of less than 0.05 was considered statistically significant in all analyses. Lower post-intervention values were expected for the EG group, so one-tailed analysis was conducted. Statistical analysis was carried out using SPSS for Windows 15.0 (SPSS, Chicago, IL).

\section{Results}

Twenty-six (26) women completed the 6-week intervention and were included in the analysis. One (1) patient in the EG group and 3 in the CG group dropped out of the study. Reasons for dropout included the following: illness, participant unable to continue due to work commitments, and 1 participant had an injury as the result of an accident in the home.

Both groups were comparable at baseline with respect to gender, age, weight, and height (all $p$ values >0.05). Additionally, at baseline there were no between-group differences in any outcome measures (all $p$-values >0.05). Table I summarizes pre- and post-intervention data for both groups.

\section{Assessment of knee extensor muscle strength}

The mean (SEM) outcome score at baseline and after the 6week intervention period is presented in Table 1. It can be seen that participants did not show any meaningful improvement in muscle strength, regardless of group allocation. While there was a slight improvement in muscle strength for the EG (1\%), the CG demonstrated a slight deterioration in muscle strength (2\%). However, the differences did not reach statistical significance. It can also be seen in Table 1 that the SEM had significantly lower values than the 
Table 1. Pre- and Postintervention Values of Both Groups for Each Outcome Measure

\begin{tabular}{|c|c|c|c|c|c|c|}
\hline & \multirow[b]{2}{*}{ Baseline p-value } & \multirow[b]{2}{*}{ SEM } & \multicolumn{2}{|c|}{$E G$} & \multicolumn{2}{|c|}{$C G$} \\
\hline & & & $T 1$ & $\mathrm{~T} 2$ & $T 1$ & $\mathrm{~T} 2$ \\
\hline \multicolumn{7}{|l|}{ Eyes open } \\
\hline Overall SI & 0.354 & 0.54 & $6.63 \pm 3.23$ & $5.5 \pm 2.67$ & $7.50 \pm 3.07$ & $6.47 \pm 2.98$ \\
\hline Mean deflection & 0.556 & 0.51 & $5.52 \pm 2.87$ & $4.66 \pm 2.55$ & $6.25 \pm 2.93$ & $5.63 \pm 2.86$ \\
\hline AP SI & 0.918 & 0.42 & $4.97 \pm 2.45$ & $4.76 \pm 2.81$ & $5.42 \pm 2.13$ & $5.33 \pm 2.68$ \\
\hline AP MD & 0.469 & 0.37 & $2.90 \pm 1.79$ & $3.60 \pm 2.81$ & $2.93 \pm 1.37$ & $3.48 \pm 2.64$ \\
\hline ML SI & 0.050 & 0.43 & $4.34 \pm 2.32$ & $2.49 \pm 1.09$ & $5.07 \pm 2.57$ & $3.35 \pm 1.97$ \\
\hline ML MD & 0.331 & 0.19 & $1.28 \pm 1.04$ & $1.17 \pm 0.75$ & $1.61 \pm 1.18$ & $1.23 \pm 0.93$ \\
\hline \multicolumn{7}{|l|}{ Eyes closed } \\
\hline Overall SI & 0.724 & 0.42 & $11.9 \pm 2.16$ & $10.7 \pm 2.64$ & $11.67 \pm 2.41$ & $11.37 \pm 2.32$ \\
\hline Mean deflection & 0.579 & 0.40 & $10.51 \pm 2.14$ & $9.26 \pm 2.57$ & $10.35 \pm 2.25$ & $9.90 \pm 2.30$ \\
\hline AP SI & 0.889 & 0.38 & $9.37 \pm 2.25$ & $8.75 \pm 2.93$ & $8.97 \pm 1.88$ & $8.86 \pm 2.40$ \\
\hline AP MD & 0.656 & 0.41 & $4.25 \pm 2.36$ & $5.01 \pm 3.71$ & $4.12 \pm 2.41$ & $3.74 \pm 2.28$ \\
\hline ML SI & 0.165 & 0.39 & $7.47 \pm 1.28$ & $5.56 \pm 1.38$ & $7.37 \pm 2.30$ & $6.25 \pm 2.05$ \\
\hline ML MD & 0.299 & 0.25 & $2.75 \pm 1.31$ & $2.01 \pm 1.34$ & $2.55 \pm 1.49$ & $2.01 \pm 1.33$ \\
\hline Muscle strength & 0.972 & 12.83 & $208.2 \pm 16.7$ & $210.7 \pm 18.1$ & $207.4 \pm 16.5$ & $202.6 \pm 13.6$ \\
\hline
\end{tabular}

SEM, standard error of mean of each variable; EG, exercise and vibration group; CG, control group; T1, pre-intervention measurement; T2, postintervention measurement; AP, anteroposterior; SI, stability index; MD, mean deflection; ML, medial-lateral.

changes obtained with training; this suggests that these changes were induced by the intervention and not related to an inherent error in the measurement.

\section{Stability assessment}

T2 It can be seen in Table 2 that the six week intervention of exercises and WBV resulted in a statistically significant improvement in MLSI for the EG over the CG. These improvements were noted when the eyes were open and when they were closed. It can also be seen that the effect size of this improvement was large with eyes closed $\left(R^{2}=0.260\right)$, and moderate when the eyes were open $\left(R^{2}=0.047\right)$. No other statistically significant difference was found.

\section{Discussion}

The aim of this study was to evaluate the effectiveness of an exercise program combined with WBV training, com- pared to an exercise program alone, in patients with FM. The exercise and WBV intervention was designed to progressively increase postural balance in standing for patients with FM. The results demonstrate that a 6-week moderateintensity exercise program, delivered twice a week, combined with supplementary WBV (3 times a week) improved patients' balance, as measured by the MLSI. This improvement was evident regardless of whether patients had their eyes open or closed.

After the intervention period participants who exercised with WBV improved postural balance (MLSI) by $42.63 \%$ with eyes open and $25.57 \%$ with eyes closed. This represents a large effect (MLSI effect size, $R^{2}=0.26$ ), when patients were evaluated with eyes closed. These improvements are similar to those reported previously ${ }^{35}$ (for example, Rees et al. ${ }^{25}$ demonstrated improvements in MLSI of $27.5 \%$ and Gusi et al. ${ }^{35}$ and Gusi et al. ${ }^{32}$ demonstrated improvements in balance of $29 \%$ and $36 \%$, respectively, with short-term WBV training).

Table 2. Between-Group Comparison of Stability with Analysis of Covariance Analysis

\begin{tabular}{|c|c|c|c|c|c|}
\hline & Degrees of freedom & $\mathrm{p}$-Value interaction & F-value & $\mathrm{p}$-Value ANCOVA & Effect size $\left(\mathrm{R}^{2}\right)$ \\
\hline $\begin{array}{l}\text { Eyes open } \\
\text { Overall SI }\end{array}$ & 28 & 0.6 & 0.47 & 0.25 & - \\
\hline Mean deflection & 28 & 0.35 & 0.47 & 0.25 & - \\
\hline AP SI & 28 & 0.8 & 0.37 & 0.43 & - \\
\hline AP MD & 28 & 0.54 & 0.47 & 0.25 & - \\
\hline ML SI & 28 & 0.1 & 3.01 & 0.045 & 0.047 \\
\hline ML MD & 28 & 0.47 & 0.007 & 0.46 & - \\
\hline \multicolumn{6}{|l|}{ Eyes closed } \\
\hline Overall SI & 28 & 0.6 & 1.79 & 0.09 & - \\
\hline Mean deflection & 28 & 0.72 & 1.76 & 0.09 & - \\
\hline AP SI & 28 & 0.94 & 0.42 & 0.26 & - \\
\hline AP MD & 28 & 0.06 & 0.64 & 0.21 & - \\
\hline ML SI & 28 & 0.97 & 12.67 & 0.0005 & 0.260 \\
\hline ML MD & 28 & 0.12 & 1.3 & 0.13 & - \\
\hline Muscle strength & 24 & 0.002 & 1.392 & 0.251 & 0.062 \\
\hline
\end{tabular}

ANCOVA, analysis of covariance; SI, stability index; AP, anteroposterior; MD, mean deflection; ML, medial-lateral; effect size, $>0.01$ small; $>0.06$ medium; $>0.14$ large. 
There were significant differences between groups when the assessment was carried out with modified visual input (i.e., participants had their eyes open or closed). Similar results were described by Thomas et al., ${ }^{13}$ who suggested that patients with FM may lack peripheral-central communication and have disturbances in vestibular signaling. Although postural balance deteriorated in both groups when participants had their eyes closed (as with the normal population $^{36}$ ), the deterioration was greater in the CG.

Improving balance with WBV intervention in those with FM is a novel and important finding, ${ }^{32}$ as it raises the possibility of using this type of training for potential health benefits beyond that normally associated with exercise in FM. For example, patients with FM have increased frequency of falls ${ }^{17}$ and may suffer premature bone loss. ${ }^{37}$ WBV can be used to address both of these important clinical problems ${ }^{35}$ in addition to improving strength and fitness in FM.

In women, decreased muscle strength is accelerated after menopause, leading to reduced function ${ }^{38}$; it has also been shown that this decrease in function can be mitigated by strengthening exercises. ${ }^{39}$ While it has been reported that WBV is associated with increased lower-limb muscle strength, ${ }^{23,27}$ because WBV also provides a strong sensory stimulus that activates the muscle spindles, it is suggested that WBV may also enhance propioception. ${ }^{40}$ The improvements achieved in muscle strength in the current study are perhaps limited because of the program duration; however, large improvements in balance were found, which may indicate that WBV training can have a relatively quick and positive influence on the proprioceptive system in FM.

It is not immediately apparent why significant differences were found in MLSI and not in APSI in this study. However, it may be the case that the kind of vibratory loading selected for this study (rotational vibration) may selectively influence Medio-Lateral Stability rather than Anterior-Posterior Stability, but this is purely speculative and further research would be necessary to test this hypothesis. Regional differences in muscle fatigue have also been reported when comparing the acute effects of rotational versus vertical vibration, ${ }^{41}$ so it is possible that muscle fatigue may have influenced outcomes in the current study also.

It has been suggested that there is a relationship between improvements in muscle strength and improved balance in people with FM. Indeed, this relationship was highlighted recently, where it was demonstrated that a 6-month exercise program resulted in statistically significant improvement in one-legged stand time for the EG over the CG. ${ }^{17}$ Although the current study did not examine the relationship between changes in muscle strength and improvements in balance, this is perhaps an area for further study.

The findings from this study are limited by small sample size and may have contributed to decreased statistical power to detect changes in some variables. The reported improvements in balance were influenced by the level of dynamic balance at baseline; therefore, greater improvements would be expected in patients with worse balance at baseline. Also, the program duration may have been too short, as previous studies based on long-term interventions demonstrated greater improvements. Another limitation of this study is that the addition of co-interventions makes it difficult to evaluate the contribution of each individual element. Further studies are needed to assess different vibratory parameters such us frequency amplitudes, time of exposure and rest, and indeed the vibratory stimuli (vertical or rotational).

\section{Conclusions}

The findings from this study are clinically important as they suggest that physical therapists should consider WBV interventions to improve balance when deficits are identified in patients with FM. The present study demonstrated that the implementation of a traditional exercise program adapted for women with FM and supplemented with WBV training leads to an improvement in MLSI. This may have implications for falls prevention in this patient group; consequently, standing postural balance should be considered a therapeutic target for patients with FM.

\section{Disclosure Statement}

No competing financial interests exist.

\section{References}

1. Wolfe F, Smythe HA, Yunus MB, et al. The American College of Rheumatology 1990 Criteria for the Classification of Fibromyalgia. Report of the Multicenter Criteria Committee. Arthritis Rheum 1990;33:160-172.

2. Martin S, Chandran A, Zografos L, et al. Evaluation of the impact of fibromyalgia on patients' sleep and the content validity of two sleep scales. Health Qual Life Outcomes 2009;7:64.

3. Mannerkorpi K, Svantesson U, Carlsson J, et al. Tests of functional limitations in fibromyalgia syndrome: A reliability study. Arthritis Care Res 1999;12:193-199.

4. Light KC, Bragdon EE, Grewen KM, et al. Adrenergic dysregulation and pain with and without acute beta-blockade in women with fibromyalgia and temporomandibular disorder. J Pain 2009;10:542-552.

5. Tomas-Carus P, Gusi N, Hakkinen A, et al. Eight months of physical training in warm water improves physical and mental health in women with fibromyalgia: A randomized controlled trial. J Rehabil Med 2008;40:248-252.

6. Brosseau L, Wells GA, Tugwell P, et al. Ottawa Panel evidence-based clinical practice guidelines for aerobic fitness exercises in the management of fibromyalgia: Part 1. Phys Ther 2008;88:857-871.

7. Alentorn-Geli E, Padilla J, Moras G, et al. Six weeks of whole-body vibration exercise improves pain and fatigue in women with fibromyalgia. J Altern Complement Med 2008; 14:975-981.

8. Busch AJ, Schachter CL, Overend TJ, et al. Exercise for fibromyalgia: A systematic review. J Rheumatol 2008;35:1130-1144.

9. Arendt-Nielsen L, Graven-Nielsen T. Muscle pain: Sensory implications and interaction with motor control. Clin J Pain 2008;24:291-298.

10. Rutledge DN, Cherry BJ, Rose DJ, et al. Do fall predictors in middle aged and older adults predict fall status in persons $50+$ with fibromyalgia? An exploratory study. Res Nurs Health 2010;33:192-206.

11. Russek LN, Fulk GD. Pilot study assessing balance in women with fibromyalgia syndrome. Physiother Theory Pract 2009;25:555-565.

12. Aydog E, Bal A, Aydog ST, et al. Evaluation of dynamic postural balance using the Biodex Stability System in rheumatoid arthritis patients. Clin Rheumatol 2006;25:462-467.

13. Thomas AW, White KP, Drost DJ, et al. A comparison of rheumatoid arthritis and fibromyalgia patients and healthy controls exposed to a pulsed (200 microT) magnetic field: 
Effects on normal standing balance. Neurosci Lett 2001; 309:17-20.

14. Hoffman DL, Dukes EM. The health status burden of people with fibromyalgia: A review of studies that assessed health status with the SF-36 or the SF-12. Int J Clin Pract 2008; 62:115-126.

15. Hauser W, Thieme K, Turk DC. Guidelines on the management of fibromyalgia syndrome: A systematic review. Eur J Pain 2010;14:5-10.

16. Tomas-Carus P, Gusi N, Häkkinen A, et al. Improvements of muscle strength predicted benefits in HRQOL and postural balance in women with fibromyalgia: An 8-month randomized controlled trial. Rheumatology (Oxford) 2009;48:1147-1151.

17. Jones KD, Horak FB, Winters-Stone K, et al. Fibromyalgia is associated with impaired balance and falls. J Clin Rheumatol 2009;15:16-21.

18. Bayazit YA, Gürsoy S, Ozer E, et al. Neurotologic manifestations of the fibromyalgia syndrome. J Neurol Sci 2002; 196:77-80.

19. Elert J, Kendall SA, Larsson B, et al. Chronic pain and difficulty in relaxing postural muscles in patients with fibromyalgia and chronic whiplash associated disorders. J Rheumatol 2001;28:1361-1368.

20. Bennett RM, Jones J, Turk DC, et al. An Internet survey of 2,596 people with fibromyalgia. BMC Musculoskelet Disord 2007;8:27.

21. Allet L, Armand S, de Bie RA, et al. The gait and balance of patients with diabetes can be improved: A randomised controlled trial. Diabetologia 2010;53:458-466.

22. Hayashibara M, Hagino $H$, Katagiri $H$, et al. Incidence and risk factors of falling in ambulatory patients with rheumatoid arthritis: A prospective 1-year study. Osteoporos Int 2010;21:1825-1833.

23. Verschueren SM, Roelants M, Delecluse C, et al. Effect of 6month whole body vibration training on hip density, muscle strength, and postural control in postmenopausal women: A randomized controlled pilot study. J Bone Miner Res 2004; 19:352-359.

24. Gillespie LD, Gillespie WJ, Robertson MC, et al. WITHDRAWN: Interventions for preventing falls in elderly people. Cochrane Database Syst Rev 2009;2:CD000340.

25. Rees SS, Murphy AJ, Watsford ML. Effects of whole body vibration on postural steadiness in an older population. J Sci Med Sport 2009;12:440-444.

26. Rees SS, Murphy AJ, Watsford ML. Effects of vibration exercise on muscle performance and mobility in an older population. J Aging Phys Act 2007;15:367-381.

27. Roelants M, Delecluse C, Verschueren SM. Whole-bodyvibration training increases knee-extension strength and speed of movement in older women. J Am Geriatr Soc 2004;52:901.

28. Cheung WH, Mok HW, Qin L, et al. High-frequency wholebody vibration improves balancing ability in elderly women. Arch Phys Med Rehabil 2007;88:852-857.

29. Bruyere O, Wuidart MA, Di Palma E, et al. Controlled whole body vibration to decrease fall risk and improve health- related quality of life of nursing home residents. Arch Phys Med Rehabil 2005;86:303-307.

30. Bautmans I, Van Hees E, Lemper JC, et al. The feasibility of Whole Body Vibration in institutionalised elderly persons and its influence on muscle performance, balance and mobility: A randomised controlled trial [ISRCTN62535013]. BMC Geriatr 2005;22:5-17.

31. Sañudo B, de Hoyo M, Carrasco L, et al. The effect of 6weeks exercise and whole body vibration on strength and quality of life in women with fibromyalgia: A randomised study. Clin Exp Rheum 2010;28(6 suppl 63):40-45.

32. Gusi N, Parraca JA, Olivares PR, et al. Tilt vibratory exercise improves the dynamic balance in fibromyalgia: A randomized controlled trial. Arthritis Care Res (Hoboken) 2010;62: 1072-1078.

33. Perron M, Hebert LJ, McFadyen BJ, et al. The ability of the Biodex Stability System to distinguish level of function in subjects with a second-degree ankle sprain. Clin Rehabil 2007;21:73-81.

34. Arnold BL, Schmitz RJ. Examination of balance measures produced by the Biodex Stability System. J Athl Train 1998; 33:323-327.

35. Gusi N, Tomas-Carus P, Hakkinen A, et al. Exercise in waisthigh warm water decreases pain and improves healthrelated quality of life and strength in the lower extremities in women with fibromyalgia. Arthritis Rheum 2006;55:66-73.

36. Lord SR, Menz HB. Visual contributions to postural stability in older adults. Gerontology 2000;46:306-310.

37. Wright SA, McVeigh JG, Finch MB. Fibromyalgia and bone health. Phys Ther Rev 2004;9:69-75.

38. Gusi N, Raimundo A, Leal A. Low-frequency vibratory exercise reduces the risk of bone fracture more than walking: A randomized controlled trial. BMC Musculoskelet Disord 2006;7:92.

39. Thomas M, Fiatarone MA, Fielding RA. Leg power in young women: Relationship to body composition, strength, and function. Med Sci Sports Exerc 1996;28:1321.

40. Nied RJ, Franklin B. Promoting and prescribing exercise for the elderly. Am Fam Physician 2002;65:419-426.

41. Stengel SV, Kemmler W, Bebenek M, et al. Effects of whole body vibration Training on Different Devices on Bone Mineral Density. Med Sci Sports Exerc 2010. [Epub ahead of print]

Address correspondence to: Borja Sañudo Corrales, PhD Department of Physical Education and Sport Faculty of Health Sciences University of Seville Pirotécnia s/n Seville E-41013 Spain

E-mail: bsancor@us.es 
WHOLE BODY VIBRATION TRAINING IN FM

Appendix. Combined Exercise Program

1. Top of page

2. Abstract

3. METHODS

4. RESULTS

5. DISCUSSION

6. Acknowledgments

7. References

8. Appendix 1. Fitness and Mobility Exercise Program Provided to Intervention Group

9. Appendix 2. Upper Extremity Program Provided to Control Group

\section{Warm-up activities (10 $\mathrm{min})$}

- Slow walking and gentle movements of the arms (e.g.: arm swinging) progressive intensity

- Tandem walking

\section{Steady-state aerobic exercise (15-20 $\mathrm{min})$}

- Brisk walking at $65 \%-70 \%$ of predicted maximum heart rate $\left(\mathrm{HR}_{\max }\right)$ (calculated as 220 - age of the participant)

- Walking in different directions (forward, backward, quick changes in direction)

- Jogging at the $65 \%-70 \%$ of predicted $\mathrm{HR}_{\max }$

* Intensity: started at $65 \%$ with increment of $10 \% \mathrm{HR}_{\max }$ every 3 weeks, as tolerated

\section{Muscle-strengthening exercises (15-20 min)}

- Each training session included eight to nine exercises: (1) shoulder press, (2) dumbbell press, (3) shoulder side elevation against resistance, (4) biceps curl, (5) dumbbell bent over row, (6) squats, (7) hip flexion and (8) extension, and (9) standing hip abductor. The different muscle groups targeted were: shoulders (deltoids and biceps), neck (trapezius), hip (gluteus and quadriceps), and back/ chest/torso (latissimus dorsi, pectoralis major, abdominals).
- Exercise progression:

- During weeks 1-3, strength training was started with one set of 8 repetitions involving the major muscle groups of the body (as outlined above) and multijoint movements. Subsequently, during weeks $3-6$, the number of repetitions was increased to 10 (one set). Participants used elastic (Thera-) bands or small weights $(1-3 \mathrm{~kg})$ as able during strength straining.

- Patients should not experience pain or excessive joint stress during or after resistance exercise

\section{Flexibility training $(10 \mathrm{~min})$}

- Flexibility exercises focused on the main areas of pain in fibromyalgia such as deltoids, biceps, neck (trapezius), hip (gluteus and quadriceps), and back/chest/torso (latissimus dorsi, pectoralis major, and abdominals).

- Stretches were static rather than ballistic.

- Participants performed eight to nine exercises: (1) Pull head to one side; (2) Posterior shoulder stretch: horizontal adduction; (3) Forearm extensor stretch: arms forward, pulling the hand with palm turned in and the fingers down; (4) Forearm flexor stretch: Place the palms against the wall and lean toward the wall; (5) Hip/gluteal stretch: flex the knee on a bench and lean toward the bench; (6) Gastrocnemius/Achilles stretch: plant one foot and keep that leg straight while leaning toward the wall; (7) Chest stretch: bend the left arm and place the forearm against the wall with the elbow at shoulder height. Rotate the body away from the arm and hold; (8) Lumbar stretch: hold the arm onto wall and lean forward; (9) Hamstring stretch: legs straight and try and touch the toes.

- Each station one set of 3 reps, holding the stretched position for $30 \mathrm{~s}$

- Exercises should be performed close to the maximum range of motion but doing this gradually to reduce the risk of exercise-induced muscle soreness and injury.

\section{Cool-down activities}

Slow walking and gentle movements of the arms 


\section{AUTHOR QUERY FOR ACM-2010-0881-VER9-CORRALES_1P}

AU1: OK to add "Faculty of Health Sciences" as in correspondence address?

AU2: minus intended after "220"? Space has been added before "age" 